\section{Prise en charge des pneumonies chez l'enfant dans les pays en voie de développement L'exemple du Malawi}

Blandine Rammaert

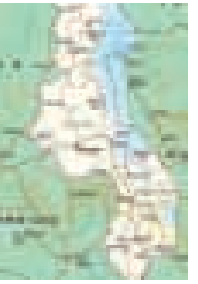

Institut Unité d'épidémiologie

et de santé publique,

Institut Pasteur du Cambodge,

5, boulevard Monivong, BP 983,

Phnom Penh, Cambodge.

brammaert@yahoo.fr

brammaert@pasteur-kh.org
Selon l'Organisation mondiale de la santé (OMS), les infections respiratoires basses, en particulier les pneumonies, sont responsables de 1,8 million de décès chez les enfants âgés de moins de 5 ans, soit $20 \%$ des décès à cet âge. Les objectifs fixés par l'OMS sont, d'ici 2015, la réduction de $25 \%$ de l'incidence des pneumonies chez les enfants de moins de 5 ans et la baisse de la mortalité de $65 \%$ par rapport à l'année 2000 [1]. Pour atteindre ces objectifs, des prises en charge standardisées ont été définies. Cependant l'application sur le terrain de ces protocoles nécessite une coordination et une coopération des autorités et des structures de santé existantes. Dans un article publié récemment, Enarson et al. présentent les résultats de la mise en place d'un programme sur 5 ans de prise en charge des pneumonies sévères chez les enfants âgés de moins de 5 ans au Malawi [2]. Le coût du projet a été en partie assuré par l'OMS et en partie par une fondation privée. Le programme comprenait la désignation d'un responsable du projet dans chaque structure de soin, des formations théoriques et pratiques du personnel médical, un recueil régulier des informations sous forme de questionnaires standardisés, le suivi des réserves de médicaments pour éviter une rupture de stock, une évaluation mensuelle et annuelle de l'impact de la prise en charge standardisée des cas de pneumonie. Les objectifs du programme ont été remplis puisque la mortalité due aux pneumonies dans la population d'enfants de moins de 5 ans a chuté de $18,6 \%$ à $8,4 \%$.
Les limites d'un tel programme ont été soulignées par les auteurs. Le premier obstacle a été le manque de personnels qualifiés qu'il a fallu former. Ensuite, les comorbidités et infections associées aux pneumonies ne sont pas toujours reconnues ni traitées correctement. Des efforts ont été faits dans ce sens. Des fonds ont également été réservés pour suppléer au manque de matériel médical et informatique. Enfin, poursuivre le programme en dehors de l'aide étrangère a représenté un réel défi. En effet, on peut s'interroger sur la pérennité de ces programmes quand il n'y a plus de supervision de la part des organismes internationaux. L'exemple du Malawi semble être un modèle de développement puisque l'expérience s'étend actuellement à d'autres centres de santé à l'intérieur du pays, 3 ans après le retrait des financements extérieurs. II faut rappeler que, malgré la solidité scientifique et technique des projets de développement, nombre d'entre eux sont voués à l'échec sans un soutien et une implication politiques. Il est également nécessaire de connaître l'épidémiologie locale avant de pouvoir mettre en place un programme de prises en charge standardisées. Au Cambodge, I'Institut Pasteur coordonne une étude épidémiologique sur les infections respiratoires basses (IRB) dans le cadre d'un projet de plus grande envergure appelé SISEA, surveillance et investigation des situations endémiques en Asie du Sud-Est. Ce projet a été financé par la communauté internationale en réponse aux épidémies de grippe aviaire et de SRAS 
(syndrome respiratoire aigu sévère). Lancée en 2007 pour une durée de 3 ans en partenariat avec 2 hôpitaux locaux, la méthodologie de l'étude sur les IRB est proche de celle décrite par Enarson et al. [2], hormis l'introduction de la prise en charge standardisée des cas. Une telle étude est un premier pas intéressant vers des actions ciblées de santé publique, car les centres choisis ont été dotés en matériel, les médecins ont reçu une formation et une relation de confiance s'est établie avec les investigateurs. Son impact et ses résultats seront connus fin 2010. Ils pourront permettre, par exemple, d'alerter les pouvoirs publics sur l'intérêt de l'introduction du vaccin anti-pneumococcique dans la prévention des pneumonies chez l'enfant et de mener à terme des actions de prise en charge standardisée. $\diamond$

Coverage of the pneumonias at the child

in developing countries, example of Malawi

\section{CONFLIT D'INTÉRÊTS}

L'auteur déclare n'avoir aucun conflit d'intérêts concernant les données publiées dans cet article.

\section{RÉFÉRENCES}

1. WHO et UNICEF. Global action plan for prevention and control pneumonia, Geneva 2009. http://www.who.int/child_adolescent_health/ documents/9789241596336/en/

2. Enarson PM, Gie R, Enarson DA, Mwansambo C. Development and implementation of a national programme for the management of severe and very severe pneumonia in children in Malawi. PLoS Med 2009; 6: e1000137.

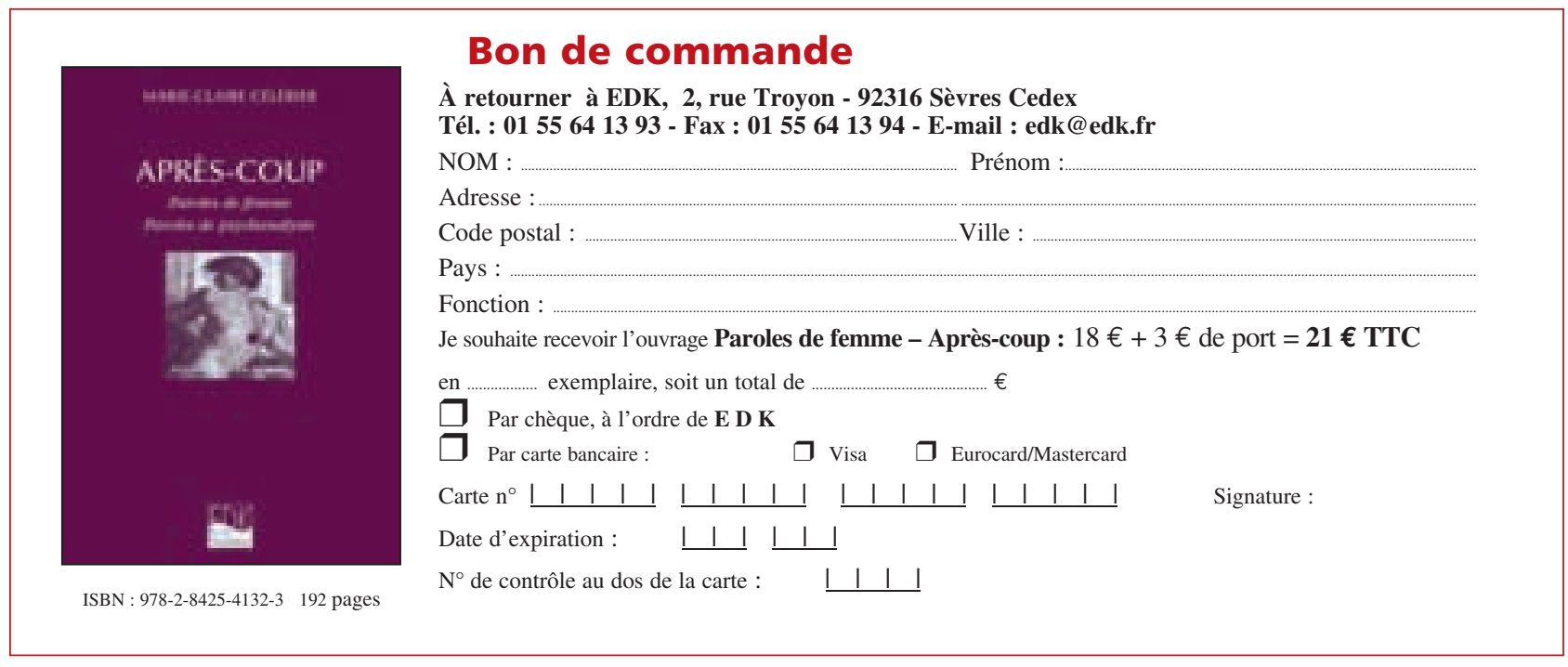

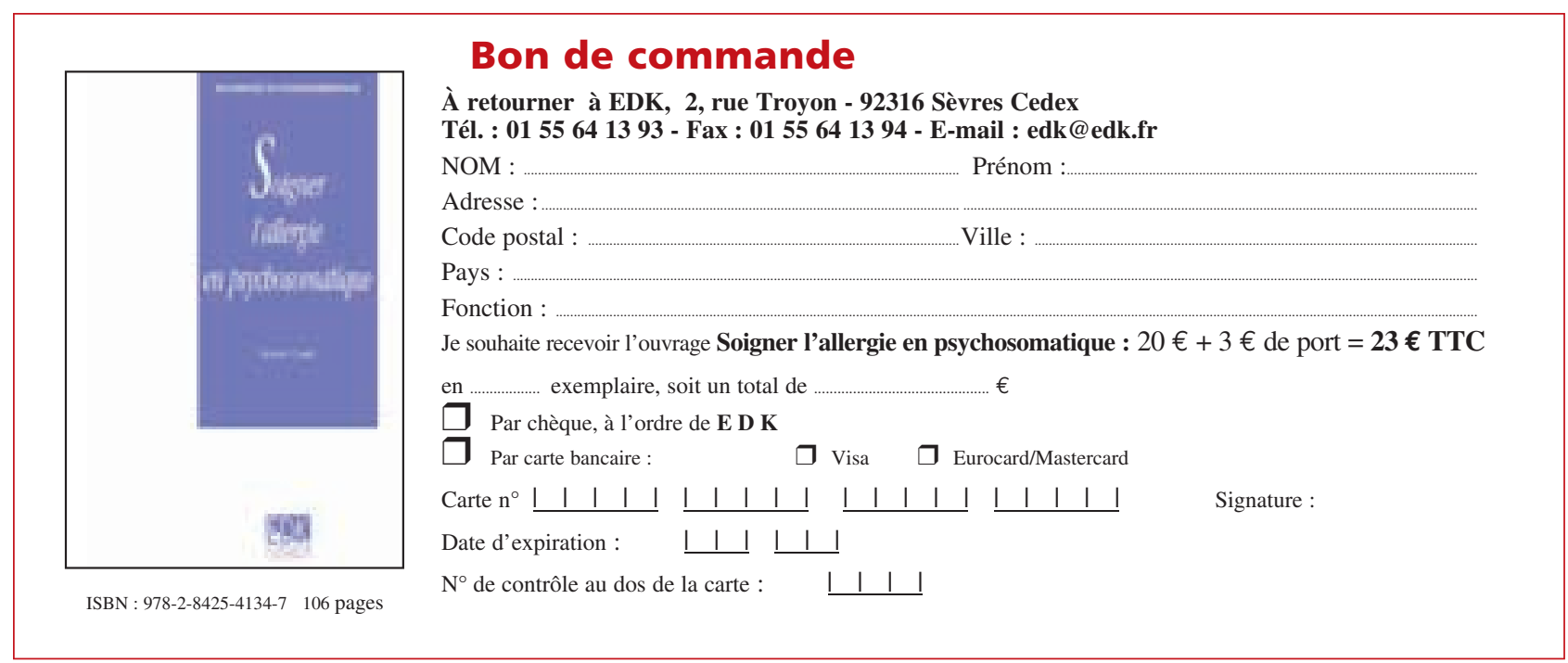

